work and extra-work accidents. The long-term effects, such as obesity, cardiovascular disease and cancer, are difficult to study, because of the need for detailed exposure assessment and the long latency periods of these diseases.

Method A review of literature on pubmed from 2000 to 2017on diet and health effects in night shift workers was conduct to collect epidemiologic evidence of diseases in night shift workers, describing their biological pathways and a set of dietary guidelines.

Results Significant Rate Ratio and Hazard Ratio increases of different diseases associated to modified eating behaviour and poor eating habits among night shift workers are reported. The night shift work is a risk factor for disruption of the circadian rhythms and for some genetic deregulation, because it produces the inversion of the sleep/wake cycle and modifies the alternation between activity and rest.

Discussion Healthy diet and improved dietary practices can reduce shift-workers' chronic disease risk. In literature was shown the importance of eating behaviour in order to prevent diseases in these workers, therefore educational programs are necessary to encourage several important lifestyle changes. Interventions to reduce chronic disease risk among shift workers should incorporate several important lifestyle changes (i.e.: healthy diet, improved dietary practices, decreased drug and alcohol use, physical activity, proper sleep and light exposure). The reported findings suggest a possible role of education programs on eating behaviour as preventive strategies in this group of workers. Actually is missing a deep knowledge of the relationship between specific dietary compositions (i.e. Mediterranean diet) and the prevention programs of diseases among shift workers.

\section{NIGHTSHIFT WORK AND PROSTATE CANCER AMONG HONG KONG CHINESE MEN}

${ }^{1}$ Lap Ah Tse ${ }^{*},{ }^{1}$ Feng Wang, ${ }^{1}$ Priscilla Ming Yi Lee, ${ }^{2}$ Wing Ming Ho, ${ }^{3}$ Chi Fai Ng. ${ }^{1}$ Division of Occupational and Environmental Health, JC School of Public Health and Primary Care, The Chinese University of Hong Kong, Hong Kong SAR, China; ${ }^{2}$ Department of Clinical Oncology, Prince of Wales Hospital, Hong Kong SAR, China; ${ }^{3} \mathrm{SH}$ Ho Urology Centre, Department of Surgery, The Chinese University of Hong Kong, Hong Kong SAR, China

\subsection{6/oemed-2018-ICOHabstracts. 1372}

Introduction A positive association between nightshift work and prostate cancer risk has been reported in epidemiological studies, but the findings have been mixed. Also, none of the previous studies has attempted to sufficiently consider the possible confounding effect from dietary sources including environmental exposure to bisphenol A (BPA). This study aims to examine the association between nightshift work and prostate cancer risk among Hong Kong Chinese men after take into account more environmental exposures.

Methods We consecutively recruited 431 incident prostate cancer cases and age frequency matched 402 controls who had complete information on nightshift work. After receiving written consents, trained researchers interviewed participants using a standard questionnaire to obtain information on socio-demographics, smoking, dietary habits, habits of using plastic food containers, family cancer history, and occupational history and nightshift work. A newly developed novel cumulative BPA exposure index (CBPAI) was used to estimate chronic BPA exposure. Odds ratio and 95\% confidence interval (95\% CI) was performed using multiple logistic regression analysis.
Results The mean age of prostate cancer cases was comparable to the controls (69.4 vs 68.2 years). Compared with the controls (39.1\%), more cases were less educated with a higher proportion of 'primary school or below' (41.1\%). More cases than controls were the nightshift workers (13.5\% vs $7.5 \%)$. After adjustment of age and socioeconomic characteristics, the OR of nightshift work to prostate cancer was 1.87 (95\% CI: 1.16 to 3.01), and the OR retained statistical significance $(\mathrm{OR}=1.76,95 \% \mathrm{CI}: 1.07$ to 2.89$)$ after environmental exposures mainly from dietary sources were further adjusted.

Conclusions Results from this study provided supportive evidence that there might be a link between nightshift work and prostate cancer. The main merit of this study is that more environmental risk factors were considered in quantifying the association.

Funding Health and Medical Research Fund (Ref. No. 11121091), Hong Kong Special Administrative Region, China.

\section{WORK SCHEDULE AND PROSPECTIVE ANTIDEPRESSANT PRESCRIPTIONS IN THE SWEDISH WORKFORCE: A 2- YEAR STUDY USING NATIONAL DRUG REGISTRY DATA}

${ }^{1} \mathrm{AL} \mathrm{Hall}{ }^{*},{ }^{2} \mathrm{G}$ Kecklund, ${ }^{2} \mathrm{C}$ Leineweber, ${ }^{2,3} \mathrm{P}$ Tucker. ${ }^{1}$ School of Population and Public Health, University of British Columbia, Vancouver, Canada; ${ }^{2}$ Stress Research Institute, Stockholm University, Stockholm, Sweden; ${ }^{3}$ Department of Psychology, Swansea University, UK

\subsection{6/oemed-2018-ICOHabstracts.1373}

Introduction Mood disorders affect millions of individuals worldwide and contribute to substantial morbidity and disability. A better understanding of modifiable work-related risk factors for depression could inform and advance prevention efforts in this area. This study used a large Swedish longitudinal occupational survey to prospectively examine the effect of self-reported work schedule on registry-based antidepressant prescriptions over a two-year period.

Methods The analytic sample $(n=8643)$ was obtained from the Swedish Longitudinal Occupational Survey of Health. Sex-stratified and unstratified analyses were conducted using logistic regression. For exposure, 8 categories were used to describe work schedule in 2008: 'regular days' ( 3 categories: night work history=none, $\leq 3$ years, or $4+$ years) 'night work (regular, rostered, or rotating)', 'regular shift work (no nights)', 'rostered work (no nights)', 'flexible/non-regulated hours', and 'other'. For the outcome, all prescriptions coded N06A according to the Anatomical Therapeutic Chemical System were obtained from the Swedish National Prescribed Drug Register and dichotomized into 'any' or 'no' prescriptions between 2008 and 2010. Estimates were adjusted for potential sociodemographic, health, and work confounders, and for prior depressive symptoms.

Results In unadjusted analyses, an increased odds ratio for depression was observed for 'Other' work hours in unstratified $(\mathrm{OR}=1.75, \quad 95 \% \mathrm{CI}: 1.21$ to 2.51$)$ and female $(\mathrm{OR}=1.62,95 \% \mathrm{CI}: 1.05$ to 2.51$)$ models; in adjusted models effects persisted but confidence intervals widened to non-significance at the $p=0.05$ level. In models adjusted for previous depressive symptoms, females in 'flexible/non-regulated' schedules showed an increased odds ratio for depression $(\mathrm{OR}=2.01,95 \% \mathrm{CI}: 1.08$ to 3.76$)$, while a decreased odds ratio was observed for the unstratified model 'regular shift work (no nights)' category ( $\mathrm{OR}=0.61 ; 95 \% \mathrm{CI}$ : 0.38 to 0.97 ). Discussion This study's findings support prospective relationships between work schedule and antidepressant prescriptions 